\title{
Oral Cachet Dosage Form
}

National Cancer Institute

\section{Source}

National Cancer Institute. Oral Cachet Dosage Form. NCI Thesaurus. Code C149365.

Solid discoid preparation consisting of a wafer enclosing a unit dose intended for oral use. 